3. Zhao, L. \& Chubb, C. F. The size-tuning of the face-distortion aftereffect. Vision Res. 41, 2979-2994 (2001).

4. Rhodes, G., Jeffery, L., Watson, T. L., Clifford, C. W. G. \& Nakayama, K. Fitting the mind to the world: Face adaptation and attractiveness aftereffects. Psychol. Sci. 14, 558-566 (2003).

5. Watson, T. L. \& Clifford, C. W. G. Pulling faces: An investigation of the face-distortion aftereffect. Perception 32, 1109-1116 (2003).

6. Moscovitch, M., Winocur, G. \& Behrmann, M. What is special about face recognition? Nineteen experiments on a person with visual object agnosia and dyslexia but normal face recognition. J. Cogn. Neurosci. 9, 555-604 (1997).

7. Farah, M. J., Wilson, K. D., Drain, M. \& Tanaka, J. N. What is "special" about face perception? Psychol Rev. 105, 482-498 (1998)

8. Kanwisher, N. Domain specificity in face perception. Nature Neurosci. 3, 759-763 (2000).

9. Diamond, R. \& Carey, S. Why faces are and are not special: An effect of expertise. J. Exp. Psychol. Gen. 115, 107-117 (1986)

10. Gauthier, I. \& Tarr, M. J. Becoming a "Greeble" expert: Exploring mechanisms for face recognition. Vision Res. 37, 1673-1682 (1997).

11. Etcoff, N. L. \& Magee, J. J. Categorical perception of facial expression. Cognition 44, 227-240 (1992).

12. Beale, J. M. \& Keil, F. C. Categorical effects in the perception of faces. Cognition 57, 217-239 (1995).

13. McKone, E., Martini, P. \& Nakayama, K. Category perception of face identity in noise isolates configural processing. J. Exp. Psychol. Hum. Percept. Perform. 27, 573-599 (2001).

14. Ekman, P. Strong evidence for universals in facial expression: A reply to Russell's mistaken critique. Psychol. Bull. 115, 268-287 (1994).

15. Young, A. et al. Facial expression megamix: tests of dimensional and category accounts of emotion recognition. Cognition 63, 271-313 (1997)

16. Levin, D. T. Race as a visual feature: Using visual search and perceptual discrimination tasks to understand face categories and the cross-race recognition deficit. J. Exp. Psychol. Gen. 129, 559-574 (2000).

17. Furl, N., Phillips, P. J. \& O'Toole, A. J. Face recognition algorithms and the other-race effect: computational mechanisms for a developmental contact hypothesis. Cogn. Sci. 26, 797-815 (2002).

18. Matsumoto, D. \& Ekman, P. Japanese and Caucasian Facial Expressions of Emotion (JACFEE) and Neutral Faces (JACNeuF) (Department of Psychology, San Francisco State Univ., San Francisco, 1988).

Acknowledgements This work was supported by the National Eye Institute.

Competing interests statement The authors declare that they have no competing financial interests.

Correspondence and requests for materials should be addressed to M.A.W. (mwebster@unr.nevada.edu).

\section{A DNA vaccine induces SARS coronavirus neutralization and protective immunity in mice}

\author{
Zhi-yong Yang ${ }^{1 \star}$, Wing-pui Kong ${ }^{1 \star}$, Yue Huang ${ }^{1}$, Anjeanette Roberts ${ }^{2}$ \\ Brian R. Murphy ${ }^{2}$, Kanta Subbarao ${ }^{2}$ \& Gary J. Nabel ${ }^{1}$
}

${ }^{1}$ Vaccine Research Center, NIAID, National Institutes of Health, Building 40, Room 4502, MSC-3005, 40 Convent Drive, Bethesda, Maryland 20892-3005, USA ${ }^{2}$ Laboratory of Infectious Diseases, NIAID, National Institutes of Health, MSC-8007, 50 South Drive, Bethesda, Maryland 20892-8007, USA

* These authors contributed equally to this work

Public health measures have successfully identified and contained outbreaks of the severe acute respiratory syndrome (SARS) coronavirus (SARS-CoV) ${ }^{1-5}$, but concerns remain over the possibility of future recurrences. Finding a vaccine for this virus therefore remains a high priority. Here, we show that a DNA vaccine encoding the spike (S) glycoprotein of the SARS$\mathrm{CoV}$ induces $\mathrm{T}$ cell and neutralizing antibody responses, as well as protective immunity, in a mouse model. Alternative forms of $S$ were analysed by DNA immunization. These expression vectors induced robust immune responses mediated by CD4 and CD8 cells, as well as significant antibody titres, measured by enzymelinked immunosorbent assay. Moreover, antibody responses in mice vaccinated with an expression vector encoding a form of $S$ that includes its transmembrane domain elicited neutralizing antibodies. Viral replication was reduced by more than six orders of magnitude in the lungs of mice vaccinated with these $S$ plasmid
DNA expression vectors, and protection was mediated by a humoral but not a T-cell-dependent immune mechanism. Gene-based vaccination for the SARS-CoV elicits effective immune responses that generate protective immunity in an animal model.

The SARS-CoV emerged in Asia as a highly aggressive pathogen that can be lethal in adults and the elderly ${ }^{1-6}$. Although its genetic organization is similar to other coronaviruses, recent phylogenetic studies suggest that it may be most closely related to type II coronaviruses $^{7-9}$. Previously described coronaviruses have not usually induced lethal disease in humans, but their pathogenicity in domestic animal species has been well documented ${ }^{10}$, and experimental vaccines developed for animals have provided insight into mechanisms of protective immunity ${ }^{11,12}$. Studies of the immune response to coronaviruses suggest that both cell-mediated and humoral immunity contribute to long-term protection ${ }^{13,14}$.

To discover the gene products and mechanisms of protective immunity relevant to SARS-CoV, two sets of cDNAs encoding the SARS-CoV S glycoproteins were prepared using modified codons to optimize expression and to minimize recombination with endogenous coronaviruses. Because coronaviruses assemble in the compartment between the endoplasmic reticulum (ER) and Golgi apparatus ${ }^{10}$, and the $S$ leader may direct it to the ER, the native leader sequence was retained in one set of vectors (Fig. 1a) and replaced in another set with a leader sequence derived from the interleukin-2 gene. Expression was not significantly altered by this leader sequence substitution (data not shown), and it was not studied further. Two S carboxy-terminal mutants, one that truncated the cytoplasmic domain $(\mathrm{S} \Delta \mathrm{CD})$ and another that deleted the transmembrane and cytoplasmic regions $(\mathrm{S} \Delta \mathrm{TM})$, were prepared, and expression of these cDNAs by a mammalian expression vector suitable for human vaccination was confirmed (Fig. 1b).

The plasmids encoding these modified S glycoproteins were analysed for their ability to elicit antiviral immunity after intramuscular injection in BALB/c mice. Injection of $S, S \Delta T M$ and $S \Delta C D$ expression vectors induced a substantial immune response. A marked increase was observed in the number of SARS-CoV S-specific CD4 Tcell immune responses (Fig. 2a), as measured by intracellular cytokine staining for interferon- $\gamma$ (IFN- $\gamma$ ) and tumour necrosis factor- $\alpha$ (TNF- $\alpha)$. In addition, substantial SARS-CoV S-specific CD8 cellular immunity was detected at levels at least sevenfold above the background response. Humoral immunity was initially

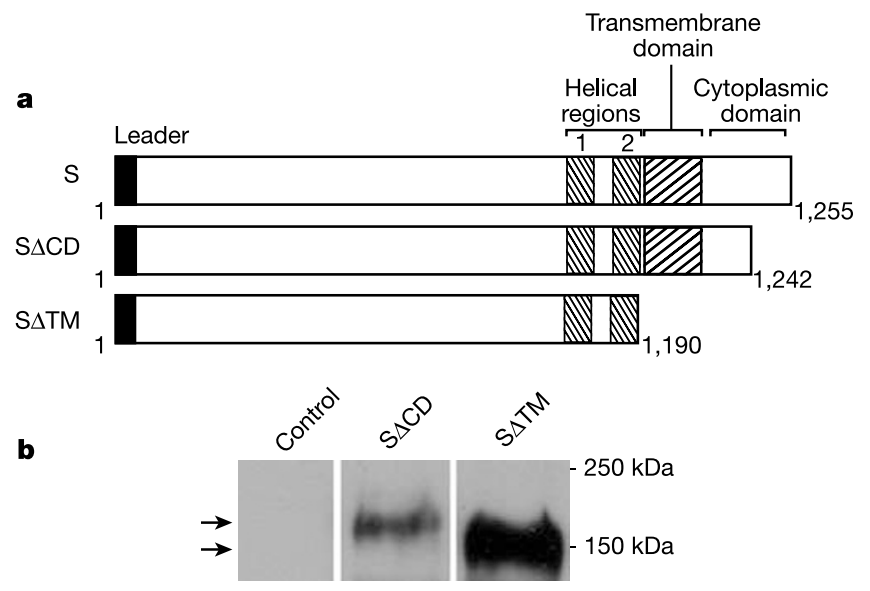

Figure 1 Schematic representation of SARS-CoV glycoprotein cDNAs and expression of recombinant proteins. a, The structure of the cDNAs used. $\mathbf{b}$, Expression of these constructs, determined by western blot analysis with antisera reactive with SARS-CoV S, was evaluated after transfection of the indicated plasmid expression vectors in 293T cells. Arrows indicate specific $\mathrm{S} \triangle \mathrm{CD}$ (upper) and $\mathrm{S} \Delta \mathrm{TM}$ (lower) bands. 
assessed using an enzyme-linked immunosorbent assay (ELISA) with lectin-captured $\mathrm{S} \Delta \mathrm{TM}$ protein expressed in 293T cells (see Methods). Substantial end-point dilution antibody titres were observed in all groups, ranging from $\sim 1: 400$ to $\sim 1: 2,000$ (Fig. 2 b, left panel).

To analyse the ability of these $S$ glycoprotein plasmids to elicit a neutralizing antibody response, a pseudotyping assay was performed $^{15}$. Animals immunized with the SARS-CoV S $\Delta C D$ expression vector induced substantial neutralizing antibody titres ranging from 1:50 to 1:150, unlike the vector control (Fig. 2b, middle panel). The $\mathrm{S}$ expression vector with the transmembrane domain deleted, $\mathrm{S} \Delta \mathrm{TM}$, also induced neutralizing antibodies, but the titres were lower (from 1:25 to 1:75). The vector with the partial cytoplasmic domain deletion induced the optimal response (Fig. 2b, right panel). Similar neutralization was observed when the complete

a

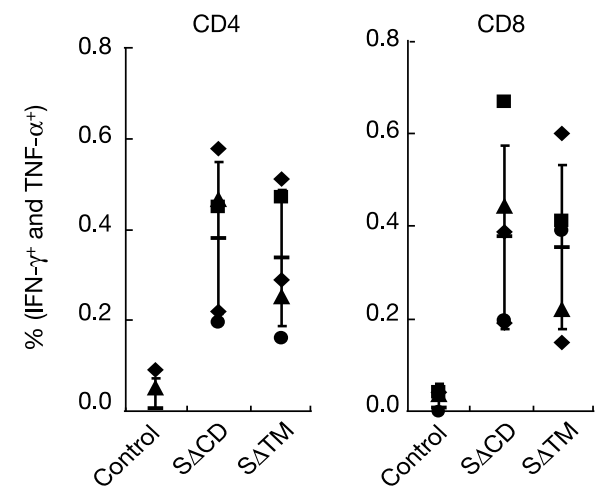

b
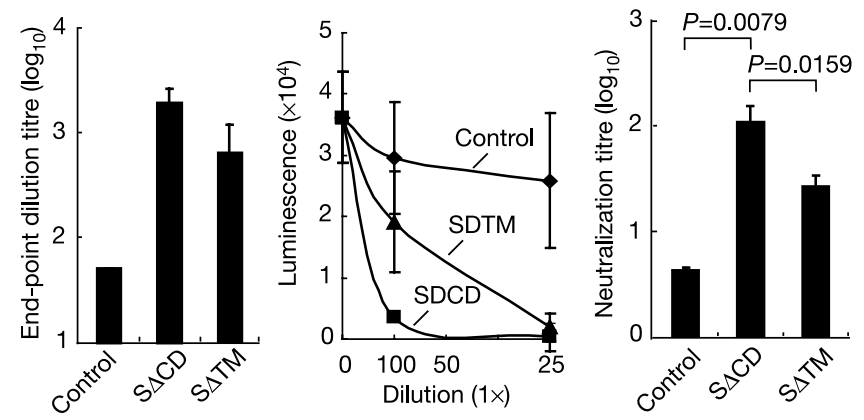

Figure 2 Immune responses to SARS-CoV DNA vaccination in BALB/C mice.

a, Intracellular cytokine staining was performed to quantify the percentage of activated T cells that produce either IFN- $\gamma$ or TNF- $\alpha$ in response to stimulation with overlapping $S$ peptide pools in CD4 (left) or CD8 (right) lymphocytes from mice ( $n=5$ per group) immunized with empty plasmid vector (control) or mice ( $n=5$ per group) immunized with the indicated plasmid at weeks 0,3 and 6 . Immune responses were measured 10 days after the final boost. Non-stimulated cells gave responses similar to those of the control subjects, at background levels. Symbols indicate the response of each individual animal, and the median value is shown (horizontal bar). $\mathbf{b}$, Antibody responses induced by plasmid DNA vaccination against the SARS-CoV S protein. End-point dilution ELISA titres of SARS-CoV S-specific antibodies (left panel) in serum of vaccinated animals collected 10 days after the final boost were determined by optical density as described in the Methods. Neutralization by antisera from mice immunized with the relevant SARS-CoV S mutant or no insert (control) plasmid DNA vectors at the indicated concentrations was measured using the luciferase assay with S pseudotyped lentiviral vectors (middle panel). Reduction of gene transfer was observed with immune sera in a dose-dependent fashion. Twofold dilutions of heat-inactivated sera were tested in a microneutralization assay for the presence of antibodies that neutralized the infectivity of 100 TCID $_{50}$ of SARS-CoV in Vero cell monolayers, using four wells per dilution on a 96-well plate (right panel). The presence of viral cytopathic effect (сре) was read on days 3 and 4 . The dilution of serum that completely prevented cpe in $50 \%$ of the wells was calculated by the Reed Muench formula. Data are presented as the mean \pm s.e. for each group. A non-parametric twotailed $t$-test (Mann-Whitney) was used for statistical analysis, and the relevant $P$ values are indicated (b, right panel).

cytoplasmic domain was removed (data not shown), suggesting that synthesis of the glycoprotein on the cell surface, without the cytoplasmic domain, is required for this response. This may be because it gives rise to a more native structure relevant to the function of this virus. Possibly, the transmembrane region helps to form a more physiological form of the $\mathrm{S}$ protein by anchoring the protein on the membrane, preserving conformational determinants and/or stabilizing the formation of the putative trimer. The same antisera were also used in a SARS-CoV microneutralization assay to assess the neutralization titre against SARS-CoV. Again, antisera from $\mathrm{S} \Delta \mathrm{CD}$-vaccinated mice elicited the most potent neutralizing antibody responses (Fig. 2b, right panel).

Infection of non-human primates with SARS-CoV has been reported, with evidence of pulmonary pathology and seroconver$\operatorname{sion}^{16}$. More recently, an animal model that may better reproduce the signs and time course of the human disease has been described. In this model, which uses ferrets, the development of pneumonitis and increased viral replication have been observed ${ }^{17}$. A murine model of SARS-CoV replication has also been described recently, in which intranasal administration of $10^{4} 50 \%$ tissue culture infectious dose $\left(\right.$ TCID $\left._{50}\right)$ units of SARS-CoV leads to virus replication in the lungs and nasal turbinates within 1-2 days ${ }^{18}$. This model afforded us the opportunity to examine immune protection against viral replication in the respiratory tract as a measure of vaccine efficacy. $\mathrm{BALB} / \mathrm{c}$ mice were immunized with the various plasmid DNAs encoding $S$ protein and challenged 30 days after the final boost. Animals were challenged intranasally with $10^{4} \mathrm{TCID}_{50}$ units of SARS-CoV (Urbani strain), and viral replication in the respiratory tract was measured 2 days later. In this analysis, the most potent immunogen, SARS S $\triangle \mathrm{CD}$, led to $>10^{6}$-fold reduction in viral load in the lungs compared with a control group injected with vector alone, in which mean viral titres of $>10^{8}$ were observed (Fig. 3a; $P=0.0079$ ). Although the $\mathrm{S}$ plasmid with the deleted transmembrane domain elicited a lower neutralizing antibody response, it was equally effective in reducing viral replication in the lungs. A 60- to 300 -fold reduction of virus titre in the nasal turbinates was also observed (Fig. 3b; $P=0.0079$ ). In both cases, evidence of pro-
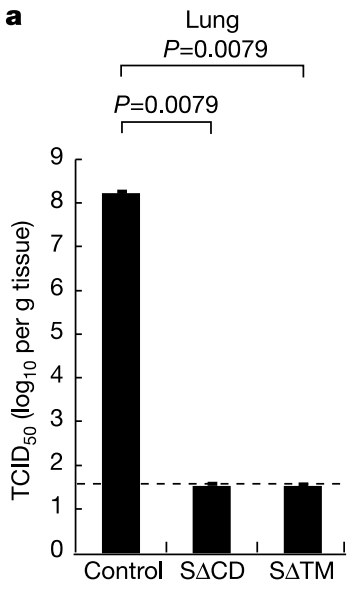

Figure 3 Protection against pulmonary SARS-CoV replication after challenge in BALB/C mice following DNA vaccination. Immunization and challenge were performed in mice as described previously ${ }^{18}$, and viral replication (mean $\log _{10} \mathrm{TCID}_{50}$ per $\mathrm{g}$ tissue with standard error) in the lower (a) and upper (b) respiratory tract after challenge with SARS-CoV was measured for five immunized animals inoculated with $\mathrm{S} \Delta \mathrm{CD}, \mathrm{S} \Delta \mathrm{TM}$ or empty plasmid vector control. The lower limit for detection of SARS-CoV replication was $1.5 \mathrm{TCID}_{50}$ per $\mathrm{g}$ in the lungs and $1.8 \mathrm{TCID}_{50}$ per $\mathrm{g}$ in the nasal turbinates. A non-parametric two-tailed $t$-test (Mann-Whitney) was used for statistical analysis. Log-transformed virus titres were compared, and statistical significance was assigned to the differences, both with a $P$ value of 0.0079 . 
ductive SARS-CoV replication was not observed in mice vaccinated with plasmid DNAs encoding $\mathrm{S} \Delta \mathrm{CD}$ or $\mathrm{S} \Delta \mathrm{TM}$.

To define the mechanism of protection, T-cell depletion with specific monoclonal antibodies was performed, and depletion in the lung and spleen was confirmed (Supplementary Fig. 1). Depletion with CD4 or CD8, alone or in combination with CD90, did not affect vaccine-induced immunity (Fig. 4a). This finding was con-

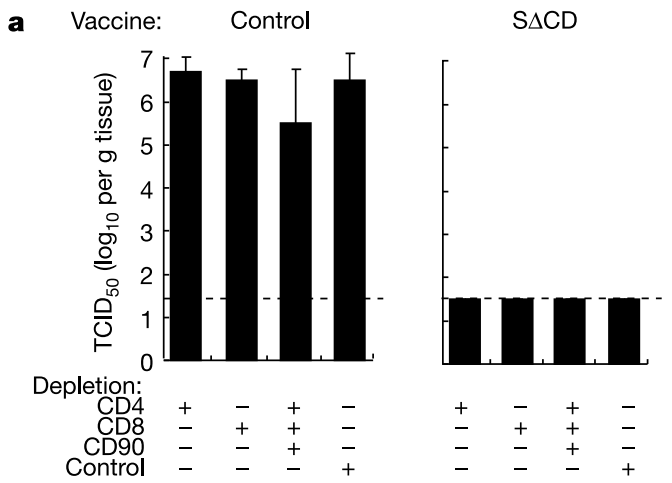

b

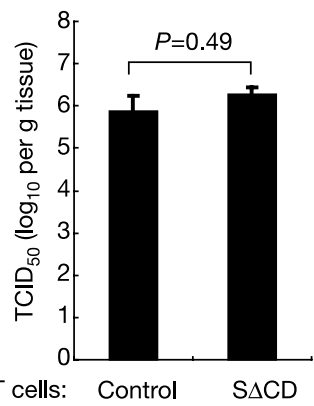

$\mathbf{C}$
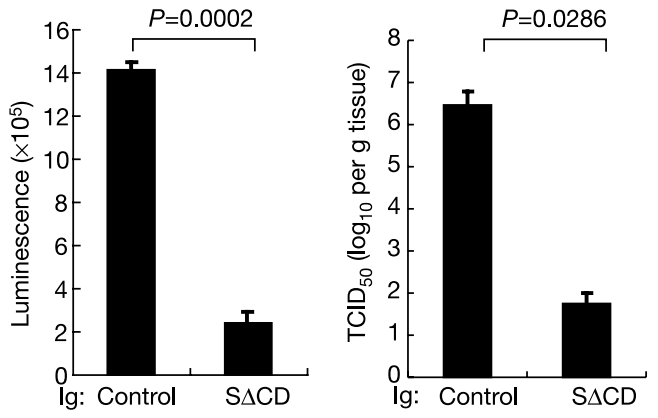

Figure 4 Immune mechanism of protection: T-cell depletion, adoptive transfer and antibody passive transfer. a, Monoclonal rat anti-mouse anti-CD4, CD8 or CD4/CD8/ CD90 were used to deplete T cells in S $\Delta \mathrm{CD}$ and control vaccinated mice $(n=4)$ as described previously ${ }^{23}$. Mice were then challenged with SARS-CoV $48 \mathrm{~h}$ later. Viral replication in the lungs was measured as described in Fig. 3. Data represent mean \pm s.e., and no statistically significant difference was observed between groups depleted as shown. $\mathbf{b}$, Lack of protection against SARS-CoV replication in the lungs after adoptive T-cell transfer from vaccinated mice. Each naive mouse $(n=4)$ received $3 \times 10^{7}$ purified T cells from a donor mouse confirmed to respond to the vaccine (immune) or from a non-immune mouse (control). The recipient mice were challenged $24 \mathrm{~h}$ after adoptive T-cell transfer. Viral titre in lungs was measured as described in Fig. 3. There was no statistically significant difference between these two groups using the non-parametric Mann-Whitney two-tailed $t$-test $(P=0.49)$. c. Protection against SARS-CoV replication in the lungs after passive transfer of immune $\lg \mathrm{G}$ from vaccinated mice. Purified IgG from $S \Delta C D$ or control vaccinated donor mice $(n=4)$ was passively transferred into recipient naive mice $(n=4)$. Serum from recipient mice was collected one day before challenge, and neutralization was confirmed using the $S$ pseudotyped lentiviral vector (left panel). Recipient mice ( $n=4$ per group) were challenged $24 \mathrm{~h}$ after IgG transfer with $10^{4} \mathrm{TCID}_{50}$ SARS-CoV and SARS-CoV replication was measured as described in Fig. 3. The non-parametric two-tailed $t$-test (Mann-Whitney) revealed a statistically significant difference of $P=0.0286$ between these two groups (right panel). firmed when the role of T-cell immunity was further analysed by adoptive $\mathrm{T}$-cell transfer; donor immune $\mathrm{T}$ cells were unable to reduce pulmonary viral replication in recipient animals (Fig. $4 \mathrm{~b}$; $P=0.49)$. By contrast, passive transfer of purified IgG from immunized, but not control, mice provided immune protection (Fig. 4c, right panel; $P=0.0286$ ) comparable to that observed in DNA-vaccinated animals (Fig. 3a). These findings indicate that immune $\mathrm{T}$ cells do not control pulmonary virus replication in this animal model, although it remains possible that $\mathrm{T}$ cells contribute to viral clearance if replication persists.

In this report, DNA vaccination has been used to induce cellular and humoral immunity to the SARS-CoV S glycoprotein. The humoral immune response includes the generation of neutralizing antibodies. This humoral immunity alone can inhibit pulmonary viral replication in a murine challenge model and suggests that DNA vaccination with the SARS-CoV S glycoprotein gene results in protective immunity. The SARS-CoV is a novel coronavirus, but vaccines for other human coronaviruses have not been successfully developed ${ }^{14}$. But considerable experience in developing vaccines against common veterinary coronaviruses has been obtained with animal coronaviruses ${ }^{11,12}$. The feline infectious peritonitis virus is a coronavirus that causes significant morbidity and mortality in domestic animals for which vaccines have been investigated, but the development of these vaccines has been complicated by possible immune potentiation of the disease and by viral evolution ${ }^{19}$. In the present study, T-cell depletion and immune IgG passive transfer were used to assess the importance of humoral responses in protection against SARS-CoV challenge. These results suggest that antibodies against SARS-CoV S glycoprotein protect against SARS-CoV challenge and do not enhance infection in this animal model. The cellular immune response appears to play a limited role in protection, although studies of animal coronaviruses have suggested that both cellular and humoral immunity contribute to protection during persistent infection ${ }^{13,14}$.

DNA vaccines have been used in a variety of animal models for different infectious diseases, including influenza, HIV, Ebola, West Nile and other viruses (reviewed in ref. 20). Despite their success in animal models, this approach has only recently been used in human studies, and its potential to protect against human diseases has yet to be established. For this reason, as well as the limitations of the animal model for SARS-CoV infections, it is important to ascertain the immunogenicity of these plasmids in humans. Should the response be suboptimal, it can be readily augmented using primeboost combinations with inactivated viral vaccine candidates or with adenoviral or poxvirus vectors. The definition of effective viral genes reported here can guide the choice of inserts for such genebased vaccination approaches. For example, the $\mathrm{S} \Delta \mathrm{CD}$ mutant can be expressed in other vector delivery systems for analysis, alone or in various combinations. Although the murine model allows assessment of various antiviral approaches and examination of the effect of immunity on pulmonary virus replication, it does not show sustained infection or high lethality. The model of infection that has been described in ferrets shows prominent pulmonary pathology associated with infection, and the efficacy of such vaccines in this and other pathogenic animal models will be of interest in future studies. At the same time, it is not known whether even the ferret model faithfully replicates the complex pathology and symptomatology of the human disease, and it will be necessary to test potential SARS vaccine candidates for their immunogenicity, safety and efficacy in humans in the event of future outbreaks.

\section{Methods}

\section{Immunogen and plasmid construction}

Plasmids encoding different versions of SARS-CoV spike (S) protein (Urbani strain, GenBank AY278741) were synthesized using human-preferred codons as previously described ${ }^{15}$. Protein expression was confirmed by western blot analysis ${ }^{21}$ with serum from a recovered patient (provided by W. Bellini). 
Vaccination

Female BALB/c mice (6-8 weeks old; Charles River Labs) were immunized with $25 \mu \mathrm{g}$ of plasmid DNA in $200 \mu \mathrm{l}$ of PBS (pH 7.4) at weeks 0, 3 and 6.

\section{Flow cytometric analysis of intracellular cytokines}

$\mathrm{CD}^{+}$and $\mathrm{CD}^{+}{ }^{+}$-cell responses were evaluated by using intracellular cytokine flow cytometry (ICC) for IFN- $\gamma$ and TNF- $\alpha$ as previously described ${ }^{21}$ with peptide pools (17-19 mers overlapping by 10 amino acids, $2.5 \mu \mathrm{g} \mathrm{ml}^{-1}$ each) covering the SARS-CoV spike protein. Cells were then fixed, permeabilized, and stained using rat monoclonal antimouse CD3, CD4, CD8, IFN- $\gamma$ and TNF- $\alpha$ (BD-Pharmingen). The IFN- $\gamma-$ and TNF- $\alpha-$ positive cells in the $\mathrm{CD} 4^{+}$and $\mathrm{CD} 8^{+}$cell populations were analysed with the program FlowJo (Tree Star, Inc.)

\section{ELISA for mouse anti-SARS-S IgG}

The mouse anti-SARS-S IgG ELISA titre was measured using a modified lectin-capture method described previously ${ }^{21}$ except the Myc-tagged, transmembrane-domaintruncated SARS-CoV S protein (SARS-S $\Delta \mathrm{TM}-\mathrm{Myc}$ ) was used for capture.

Inhibition of viral gene transfer to measure mouse antibody titre

SARS-CoV spike pseudotyped lentiviruses expressing a luciferase reporter gene were produced by transfecting $293 \mathrm{~T}$ cells with the following plasmids: $7 \mu \mathrm{g}$ of pCMV $\Delta \mathrm{R} 8.2,7 \mu \mathrm{g}$ of pHR'CMV-Luc and $400 \mathrm{ng}$ CMV/R-SARS-S. Cells were transfected overnight, washed and replenished with fresh media. Forty-eight hours later, supernatants were harvested, filtered through a $0.45 \mu \mathrm{m}$ syringe filter, aliquotted and used immediately or frozen at $-80^{\circ} \mathrm{C}$. p24 levels were measured from different viral stocks using the Coulter HIV-1 p24 Antigen Assay kit (Beckman Coulter). Antisera were mixed with $100 \mu \mathrm{l}$ of pseudoviruses a various dilutions and added to Vero cells in 48 -well dishes (30,000 cells per well). Plates were washed and fresh media were added $14-16 \mathrm{~h}$ later. Forty-eight hours after infection, cells were lysed in mammalian cell lysis buffer (Promega). A standard quantity of cell lysate was used in a luciferase assay with luciferase assay reagent (Promega) according to the manufacturer's protocol.

\section{Neutralization of SARS-CoV by mouse immune antisera}

Twofold dilutions of heat-inactivated sera were tested in a microneutralization assay for the presence of antibodies that neutralized the infectivity of $100 \mathrm{TCID}_{50}$ of SARS-CoV on Vero cell monolayers, using four wells per dilution on a 96-well plate. The presence of viral cytopathic effect (cpe) was tested for on days 3 and 4 . The dilution of serum that completely prevented cpe in $50 \%$ of the wells was calculated by the Reed Muench formula ${ }^{22}$.

\section{Challenge of immunized mice with SARS-CoV}

Vaccinated mice were lightly anaesthetized with isoflurane and inoculated with $50 \mu \mathrm{l}$ of diluted virus $\left(10^{4} \mathrm{TCID}_{50}\right.$ of SARS-CoV; Urbani strain) intranasally according to institutional animal care and use guidelines in an ABSL3 facility. On day 2, mice were euthanized and lungs and nasal turbinates were removed and stored at $-70{ }^{\circ} \mathrm{C}$ until the end of the study. The frozen tissues were thawed and homogenized in a $10 \%$ (lungs) or $5 \%$ (nasal turbinates) w/v suspension in Leibovitz 15 medium (Invitrogen), and virus titres were determined in Vero cell monolayers in 24- and 96-well plates. Virus titres are expressed as TCID $_{50}$ per $g$ of tissue. The lower limit of detection of infectious virus in $10 \%$ and $5 \% \mathrm{w} / \mathrm{v}$ suspensions is 1.5 for lung and $1.8 \log _{10} \mathrm{TCID}_{50}$ per g for nasal turbinate homogenates, respectively.

\section{Depletion of T-cell subsets in vivo}

To deplete specific T-cell subsets, known rat monoclonal antibodies (anti-mouse CD4 (GK1.5), anti-mouse CD8(2.43) or anti-mouse CD90(30-H12)), prepared as described previously $y^{23}$ and provided by S. L. Epstein (FDA), were administered intraperitoneally (1 mg each in $1 \mathrm{ml}$ PBS) $48 \mathrm{~h}$ before challenge. The depletion was confirmed before challenge (Supplementary Fig. 1).

\section{Passive transfer of immunoglobin}

IgG from mice immunized with plasmid DNA encoding S $\triangle \mathrm{CD}$ (immune) or no insert (controls) was purified from sera using a Protein A Antibody Purification Kit (Sigma), and neutralization activity was confirmed using the mouse pseudotyping assay. Briefly, $0.3 \mathrm{ml}$ of purified IgG (from approximately $1 \mathrm{ml}$ of serum) was administered intravenously into each recipient naive mouse ( $n=4$ per group) by tail vein injection $24 \mathrm{~h}$ before challenge. Sera were collected from recipient BALB/c mice $3 \mathrm{~h}$ post transfer to confirm the neutralizing antibody titre in recipient animals.

\section{Adoptive T-cell transfer studies}

$\mathrm{T}$ cells from vaccinated (immune) or nonimmune (control) mice were enriched using a Pan T-Cell Isolation Kit (Miltenyi Biotec). Approximately $3 \times 10^{7} \mathrm{~T}$ cells in $0.5 \mathrm{ml}$ PBS were administered into each recipient naive $\mathrm{BALB} / \mathrm{c}$ mouse intravenously through the tail vein $24 \mathrm{~h}$ before challenge. There were four recipient mice per group.

Received 9 February; accepted 8 March 2004; doi:10.1038/nature02463.

Ksiazek, T. G. et al. A novel coronavirus associated with severe acute respiratory syndrome. $N$. Engl. J. Med. 348, 1953-1966 (2003).

2. Drosten, C. et al. Identification of a novel coronavirus in patients with severe acute respiratory syndrome. N. Engl. J. Med. 348, 1967-1976 (2003).

3. Tsang, K. W. et al. A cluster of cases of severe acute respiratory syndrome in Hong Kong. N. Engl. J. Med. 348, 1977-1985 (2003).

4. Lee, N. et al. A major outbreak of severe acute respiratory syndrome in Hong Kong. N. Engl. J. Med. 348, 1986-1994 (2003)
5. Poutanen, S. M. et al. Identification of severe acute respiratory syndrome in Canada. N. Engl. J. Med. 348, 1995-2005 (2003).

6. World Health Organization. Consensus document on the epidemiology of severe acute respiratory syndrome (SARS). WHO $\langle\mathrm{http}: / /$ www.who.int/csr/sars/en/WHOconcensus.pdf $\rangle$ (2003).

7. Rota, P. A. et al. Characterization of a novel coronavirus associated with severe acute respiratory syndrome. Science 300, 1394-1399 (2003).

8. Marra, M. A. et al. The genome sequence of the SARS-associated coronavirus. Science 300, 1399-1404 (2003)

9. Snijder, E. J. et al. Unique and conserved features of genome and proteome of SARS-coronavirus, an early split-off from the coronavirus group 2 lineage. J. Mol. Biol. 331, 991-1004 (2003).

10. Lai, M. M. C. \& Holmes, K. V. in Fields Virology (ed. Knipe, D. M.) 1163-1185 (Lippincott, Williams \& Wilkins, Philadelphia, 2001).

11. Saif, L. J. Coronavirus immunogens. Vet. Microbiol. 37, 285-297 (1993).

12. Park, S. et al. Immune response of sows vaccinated with attenuated transmissible gastroenteritis virus (TGEV) and recombinant TGEV spike protein vaccines and protection of their suckling pigs against virulent TGEV challenge exposure. Am. J. Vet. Res. 59, 1002-1008 (1998).

13. Sestak, K. et al. Active immunity and T-cell populations in pigs intraperitoneally inoculated with baculovirus-expressed transmissible gastroenteritis virus structural proteins. Vet. Immunol. Immunopathol. 70, 203-221 (1999).

14. Holmes, K. V. in Fields Virology (ed. Knipe, D. M.) 1187-1203 (Lippincott Williams \& Wilkins, Philadelphia, 2001).

15. Yang, Z.-Y. et al. pH-dependent entry of SARS coronavirus is mediated by the Spike glycoprotein and enhanced by dendritic cell-mediated transfer through DC-SIGN. J. Virol. (in the press).

16. Fouchier, R. A. et al. Aetiology: Koch's postulates fulfilled for SARS virus. Nature 423, 240 (2003)

17. Martina, B. E. et al. Virology: SARS virus infection of cats and ferrets. Nature 425, 915 (2003).

18. Subbarao, K. et al. Prior infection and passive transfer of neutralizing antibody prevent replication of SARS coronavirus in the respiratory tract of mice. J. Virol. 78, 3572-3577 (2004).

19. Perlman, S. Pathogenesis of coronavirus-induced infections. Review of pathological and immunological aspects. Adv. Exp. Med. Biol. 440, 503-513 (1998).

20. Gurunathan, S., Klinman, D. M. \& Seder, R. A. DNA vaccines: immunology, application, and optimization. Annu. Rev. Immunol. 18, 927-974 (2000).

21. Kong, W. P. et al. Immunogenicity of multiple gene and clade human immunodeficiency virus type 1 DNA vaccines. J. Virol. 77, 12764-12772 (2003).

22. Reed, L. J. \& Muench, H. A simple method of estimating fifty percent endpoints. Am. J. Hyg. 27, 493-497 (1938).

23. Epstein, S. L. et al. Vaccination with DNA encoding internal proteins of influenza virus does not require $\mathrm{CD} 8(+)$ cytotoxic T lymphocytes: either $\mathrm{CD} 4(+)$ or $\mathrm{CD} 8(+) \mathrm{T}$ cells can promote survival and recovery after challenge. Int. Immunol. 12, 91-101 (2000).

Supplementary Information accompanies the paper on www.nature.com/nature

Acknowledgements We thank J. McAuliffe and L. Vogel from the Laboratory of Infectious Diseases, M. St Claire, T. Tobery and staff at Bioqual for assistance in challenge studies, S. Rao and his staff for assistance in passive and adoptive transfer studies, J. Mascola for discussion, S. Epstein for supplying the monoclonal antibodies, A. Tislerics and T. Suhana for manuscript preparation, K. Stroud for preparation of figures, and members of the Nabel laboratory for advice and discussions.

Competing interests statement The authors declare that they have no competing financial interests.

Correspondence and requests for materials should be addressed to G.J.N. (gnabel@nih.gov)

\section{Differentiating germ cells can revert into functional stem cells in Drosophila melanogaster ovaries}

\section{Toshie Kai \& Allan Spradling}

Howard Hughes Medical Institute Research Laboratories, Department of Embryology, Carnegie Institution of Washington, 115 W. University Parkway, Baltimore, Maryland 21210, USA

Many tissues including blood, skin, gut and germ cells are continuously maintained by tissue stem cells ${ }^{1-2}$. Under certain conditions, however, other organs can undergo repair using stem-cell-like progenitors generated by cell de-differentiation ${ }^{3}$. Cell fates have been broadened experimentally ${ }^{4-7}$, but mechanisms allowing de-differentiation to a stem cell state are poorly known. Germline stem cells begin to differentiate by forming interconnected germ cell cysts (cystocytes), and under certain 